\title{
Optimal Asset Allocation with Heterogeneous Persistence of Shocks *
}

\author{
Domenica Di Virgilio* Fulvio Ortu ${ }^{* *}$ Federico Severino ${ }^{* * *}$ \\ Claudio Tebaldi ${ }^{* * * *}$ \\ *Bank of Slovenia (e-mail: domdivirgilio@gmail.com). \\ ** Università Bocconi and IGIER, (e-mail: fulvio.ortu@unibocconi.it) \\ *** Università della Svizzera Italiana, SFI and Università Bocconi \\ (e-mail: federico.severino@usi.ch) \\ **** Università Bocconi and IGIER, (e-mail: \\ claudio.tebaldi@unibocconi.it)
}

Keywords: optimal portfolio, Epstein-Zin preferences, multiple horizons, persistence of returns, shocks heterogeneity.

\section{INTRODUCTION}

There is wide evidence that financial time series are the outcome of the superposition of processes with heterogeneous frequencies. This is true, in particular, for market return. Indeed, log market return can be decomposed into uncorrelated components that explain the reaction to shocks with different persistence. The instrument that allows us to do so is the Extended Wold Decomposition of Ortu, Severino, Tamoni and Tebaldi (2017). Hence, we construct portfolios of these components in order to maximize the utility of an agent with a fixed investment horizon. In particular, we build upon Campbell and Viceira (1999) solution of the optimal consumption-investment problem with Epstein-Zin utility, by using a rebalancing interval of $2^{J}$ periods. It comes out that the optimal asset allocation involves all the persistent components of market $\log$ return up to scale $J$. Such components play a fundamental role in characterizing both the myopic and the intertemporal hedging demand. Moreover, the optimal policy prescribes an increasing allocation on more persistent assets when the investor's relative risk aversion rises. Finally, portfolio reallocation every $2^{J}$ periods is consistent with rational inattention. Indeed, observing assets value is costly and transaction costs make occasional rebalancing optimal.

\section{SUMMARY}

Given a zero-mean weakly stationary time series $\mathbf{x}=$ $\left\{x_{t}\right\}_{t}$, the Classical Wold Decomposition allows us to write any $x_{t}$ as an infinite sum of uncorrelated innovations:

$$
x_{t}=\sum_{k=0}^{\infty} \alpha_{h} \varepsilon_{t-h}
$$

where $\varepsilon=\left\{\varepsilon_{t}\right\}_{t}$ is a unit variance white noise and $\alpha_{h}$ are the so-called impulse response functions. The Extended Wold Decomposition introduced by Ortu, Severino, Tamoni and Tebaldi (2017), instead, decomposes $x_{t}$ into

\footnotetext{
* This abstract is for the Minisymposium Dynamic models in economics and management.
}

uncorrelated persistent components $x_{t}^{(j)}$ associated with specific scales $j$ :

$$
x_{t}=\sum_{j=1}^{+\infty} x_{t}^{(j)}, \quad x_{t}^{(j)}=\sum_{k=0}^{+\infty} \beta_{k}^{(j)} \varepsilon_{t-k 2^{j}}^{(j)} .
$$

Here each detail process $\varepsilon^{(j)}=\left\{\varepsilon_{t}^{(j)}\right\}_{t}$ is an $M A\left(2^{j}-1\right)$ with respect to the fundamental innovations of $\mathbf{x}$ and $\beta_{k}^{(j)}$ is the multiscale impulse response associated with scale $j$ and time-shift $k 2^{j}$. Specifically,

$$
\varepsilon_{t}^{(j)}=\frac{1}{\sqrt{2^{j}}}\left(\sum_{i=0}^{2^{j-1}-1} \varepsilon_{t-i}-\sum_{i=0}^{2^{j-1}-1} \varepsilon_{t-2^{j-1}-i}\right)
$$

and

$$
\beta_{k}^{(j)}=\frac{1}{\sqrt{2^{j}}}\left(\sum_{i=0}^{2^{j-1}-1} \alpha_{k 2^{j}+i}-\sum_{i=0}^{2^{j-1}-1} \alpha_{k 2^{j}+2^{j-1}+i}\right)
$$

for any $j \in \mathbb{N}$ and $k \in \mathbb{N}_{0}$. Moreover, fixed a maximum scale $J$, it is possible to write the orthogonal decomposition

$$
x_{t}=\sum_{j=1}^{J} x_{t}^{(j)}+m_{t}^{(J)}
$$

where $m_{t}^{(J)}$ constitutes a residual component. With a small abuse of notation we denote $x_{t}^{(J+1)}=m_{t}^{(J)}$. The support $\left\{t-k 2^{j}\right\}$ of details $\varepsilon_{t}^{(j)}$ is sparser and sparser as the scale raises, conveying the intuition of increasing duration, together with the higher order of $M A$. Therefore, scale-specific impulse responses $\beta_{k}^{(j)}$ capture the sensitivity of $x_{t}$ with respect to underlying shocks with increasing durations of $2,4,8, \ldots$ periods.

We apply the previous decomposition to the process of market log returns associated, for instance, to S\&P 500 index. We consider an Epstein-Zin investor that chooses how to distribute her wealth among $J+1$ risky assets and a riskless security, with a periodic rebalancing every $2^{J}$ periods. Log returns of these risky assets are supposed to mimic the persistent components $x_{t}^{(1)}, \ldots, x_{t}^{(J+1)}$ of 
market log returns. Moreover, each $x_{t}^{(j)}$ is assumed to follow an $A R(1)$ process on its own scale:

$$
x_{t+2^{j}}^{(j)}=\mu_{j}\left(1-\phi_{j}\right)+\phi_{j} x_{t}^{(j)}+s_{j} \varepsilon_{t+2^{j}}^{(j)} .
$$

Finally, the fundamental innovations $\varepsilon_{t}$ are i.i.d. and distributed as standard normal.

By denoting portfolio loadings by $\pi_{t}(j)$, the return over $2^{J}$ periods is, then,

$$
\begin{aligned}
R_{p, t+2^{J}}= & \sum_{j=1}^{J+1} \pi_{t}(j) e^{x_{t+2^{J}}(j)} \\
& +\left(1-\sum_{j=1}^{J+1} \pi_{t}(j)\right) e^{2^{J} r_{f}} .
\end{aligned}
$$

The previous assumptions allow the vector of returns $\mathbf{z}_{t}=\left[x_{t}^{(1)}, \ldots, x_{t}^{(J+1)}\right]^{\prime}$ to follow the $V A R$ dynamics

$$
\mathbf{z}_{t+2^{J}}=\Phi_{0}+\Phi \mathbf{z}_{t}+\mathbf{v}_{t+2^{J}},
$$

where $\Phi_{0}$ is a vector, $\Phi$ is a diagonal matrix and $\mathbf{v}_{t}$ is a multivariate white noise on the time grid $\left\{t-k 2^{J}\right\}$ with $k \in \mathbb{Z}$. The orthogonality properties of the Extended Wold Decomposition are crucial for obtaining a white noise here.

Our investor has recursive preferences but her utility depends on the current consumption and the certainty equivalent associated with the utility $2^{J}$ periods ahead:

$$
\begin{gathered}
\max _{\left\{C_{t}, \boldsymbol{\pi}_{t}\right\}_{t=2^{J} J}} U_{t}=\left((1-\beta) C_{t}^{(1-\gamma) / \theta}+\beta E_{t}\left[U_{t+2^{J}}^{1-\gamma}\right]^{1 / \theta}\right)^{\theta /(1-\gamma)}[1] \\
\text { sub } \\
W_{t+2^{J}}=R_{p, t+2^{J}}\left(W_{t}-C_{t}\right)
\end{gathered}
$$

where $0<\beta<1$ is the preference discount factor, $\gamma>0$ is the coefficient of relative risk aversion, $\psi$ denotes the intertemporal elasticity of substitution (IES) and $\theta=$ $(1-\gamma) /\left(1-\psi^{-1}\right)$. Consumption $C_{t}$ and wealth $W_{t}$ are scalars, while the vector $\boldsymbol{\pi}_{t}$ contains portfolio weights associated with the $J+1$ securities into consideration.

The previous VAR representation of returns allows us to embed our optimal consumption-investment problem into Campbell, Chan and Viceira (2003) strategic allocation theory. In particular, we exploit the affine guess

$$
\boldsymbol{\pi}_{t}=A_{0}+A_{1} \mathbf{z}_{t},
$$

paired with the quadratic log consumption-wealth ratio

$$
c_{t}-w_{t}=b_{0}+B_{1}^{\prime} \mathbf{z}_{t}+\mathbf{z}_{t}^{\prime} B_{2} \mathbf{z}_{t},
$$

where $b_{0}$ is a scalar, $A_{0}$ and $B_{1}$ are vectors and $B_{2}$ is a square matrix.

After approximating log return, budget constraint and Euler equation following the standard derivation with pace $2^{J}$, we determine the approximate optimal asset allocation, which is driven by myopic and hedging motives:

$$
\begin{aligned}
\boldsymbol{\pi}_{t}= & A_{0, \text { myopic }}+A_{0, \text { hedging }} \\
& +\left(A_{1, \text { myopic }}+A_{1, \text { hedging }}\right) \mathbf{z}_{t} .
\end{aligned}
$$

The orthogonality of the Extended Wold Decomposition ensures that the myopic part of $\pi_{t}(j)$ depends only on $x_{t}^{(j)}$. Moreover, if $\gamma=1$ - because, for instance, the investor has logarithmic utility - the hedging part of $\boldsymbol{\pi}_{t}$ disappears. Then, for a myopic investor the weight $\pi_{t}(j)$ depends only on $x_{t}^{(j)}$.
Instead, if $\gamma \neq 1$, the resulting capital allocation on the $j$-th component of market returns depends also on the other components. In particular, $\pi_{t}(j)$ depends on $x_{t}^{(i)}$ with $i \neq j$ through the term $A_{1, \text { hedging }} \mathbf{z}_{t}$. Hence, the share $\pi_{t}(j)$ invested in the component $x_{t}^{(j)}$ depends on the components at scales $i \neq j$ just for hedging purposes.

Finally, although the investor's horizon is $2^{J}$, the optimal capital allocation involves all the components of market returns, not only the one at scale $J$.

Once $A_{0}$ and $A_{1}$ are determined, we assess that the log consumption-wealth ratio is actually quadratic in the state vector $\mathbf{z}_{t}$ and we are able to find the optimal $b_{0}, B_{1}$ and $B_{2}$.

We corroborate our analysis by estimating optimal weights of a portfolio investing in persistent components of S\&P 500 index. By employing daily data, we can capture the impact of two-day shocks on time scale $j=1$, (roughly) weekly shocks on scale $j=2$ and so on and so forth. Moreover, we consider investors with different values of risk aversions. If $\gamma=1$ the agent is fully myopic and the weights are all equal across scales. When $\gamma$ increases, instead, the investor diversifies within persistent assets and portfolio loadings to high scales become prominent.

\section{REFERENCES}

Campbell J.Y., Chan Y.L, Viceira L.M. (2003), "A multivariate model of strategic asset allocation", Journal of financial economics, 67, 1, 41-80.

[2] Campbell J.Y., Viceira L.M. (1999), "Consumption and portfolio decisions when expected returns are time varying", Quarterly Journal of Economics, 114, 433-495.

[3] Ortu F., Severino F., Tamoni A., Tebaldi C. (2017), "A persistence-based Wold-type decomposition for stationary time series", Available at https : //papers.ssrn.com/sol3/papers.cfm?abstract ${ }_{i} d=$ 1973049 . 